\title{
A Special Issue on Petroleum Geostatistics
}

\author{
Leonardo Azevedo' ${ }^{1}$ Jo Eidsvik ${ }^{2}$
}

Published online: 25 March 2021

(C) International Association for Mathematical Geosciences 2021

In September 2019, about 170 geoscientists met in the birthplace of the Renaissance for the Fourth European Association of Geoscientists and Engineers Conference on Petroleum Geostatistics. This was the first meeting with a 4-year time interval, following the previous 2015 gathering in beautiful Biarritz. The conference showed, once again, the vitality of our community. The conference lasted for five days and covered a wide range of topics, such as the most recent advances on geostatistical modeling tools of continuous and categorical subsurface properties, stochastic geophysical and production inversion methods and the new trends on spatial data sciences, including deep learning and its integration in the geo-modeling workflow. The works presented in the meeting sparked many interesting discussions at the end of each day.

Following this conference, we invited paper submissions for a special issue on petroleum geostatistics in Mathematical Geosciences. Most of the papers in this issue were presented at the conference in Florence, Italy, but participation there was not a requirement for neither submission nor acceptance. The special issue includes seven papers on new modeling approaches, methodologies and applications in the field of geostatistics for the petroleum industry.

Petroleum geostatistics has strong traditions in geo-modeling, as well as in the integration of various data sources and uncertainty quantification of spatially dependent

\footnotetext{
$凶$ Leonardo Azevedo

leonardo.azevedo@tecnico.ulisboa.pt

Jo Eidsvik

jo.eidsvik@ntnu.no

1 CERENA, Instituto Superior Técnico, Lisboa, Portugal

2 NTNU, Trondheim, Norway
} 
reservoir variables. These elements are clearly reflected in the contributions of this special issue. The field has broadened over the last few years, building upon recent developments in machine learning and spatial data science. In this vein, several of the special issue papers use ensemble realizations to represent the reservoir uncertainties or build on computationally efficient lower-dimensional reservoir model representations to mimic the core elements required for reliable reservoir predictions with associated uncertainties.

We next discuss each of the special issue papers in alphabetical order:

Ismagilov et al. present a new approach to simulate categorical reservoir properties with two facies using Boolean functions. They show how Boolean functions can be trained from well data to be decomposed into a binary version of Fourier series. Their approach enables the fast simulation of reservoir facies in three-dimensional space and is capable of accounting for vertical non-stationarity. A suggested workflow is tested on a real-world oil field, and the new method reproduces statistical patterns from the well data better than the standard sequential indicator simulation method.

Loe et al. present an approach for reservoir monitoring based on ensemble-based methods for mixed discrete-continuous reservoir variables. They use inverted subsurface properties predicted from time-lapse geophysical data to constrain the fluid front in a two-phase water/hydrocarbon reservoir flow problem. A synthetic two-dimensional reservoir simulation example is used to demonstrate the approach. The results show the potential of the method in predicting the true location of the binary states of fluid facies. Controlled-source electromagnetic resistivity data are used as the geophysical time-lapse information.

Moyen and Gentilhomme present adaptive ensemble-based optimization methods for petrophysical inversion. They develop an optimization algorithm that is designed for sampling the solution space of nonlinear, non-convex quadratic problems, and this is embedded in an ensemble-based optimization method. The method is applied to a real-world oil field, where porosity, shale fraction and fluid saturations are inverted under continuity and Lagrange constraints, which are conditioned by P-impedance seismic data. Results show that the quality of the geological models is improved from earlier analyses.

Stordal et al. present the Stein variational gradient descent method within ensemble settings to solve history matching problems. The approach is modified to handle p-kernels that replace Gaussian kernels. This allows one to alleviate some a priori assumptions about the model parameters. The experimental results show the ability of using p-kernels in high-dimension problems. The proposed method is illustrated in chaotic dynamical systems and then applied to a reservoir example. Here the results show a better performance of p-kernels in uncertainty estimation than the natural competitor using Gaussian kernels.

Talarico et al. present new results obtained for predicting the spatial configuration of geological facies using recursive neural networks and convolutional hidden Markov models from seismic amplitude data. The proposed inversion technique is validated using synthetic seismic data in the context of a complex geological environment. Their synthetic example demonstrates that the suggested method can facilitate geologically 
realistic predictions of facies, borrowing beneficial elements from both Markovian modeling and neural network models.

Walsh and Manzocchi present pixel-based modeling techniques that produce realistic high net-to-gross ratio models with low connectivity of the net facies, a common combination in many natural geological systems. To model this challenging sedimentary environment efficiently, the authors build on a geometrical compression method. They base this construction on percolation theory and challenges related to the coupling of net-to-gross ratio and connectivity. An application shows realistic facies models with quantifiable levels of connectivity that are defined independently of the model net-to-gross ratio.

Yu et al. present a decorrelation technique between elastic model parameters that is applicable to Bayesian linearized inversion of pre-stack seismic data. They address the challenge of reliably linking cross-variograms established from sparse well-log data in the seismic inversion step. The decorrelation method utilizes principal component analysis, and the technique entails that the cross-variograms between the three independent model parameters are zero. Synthetic and real application examples illustrate the benefit of the suggested method compared with the state of the art. 